\title{
MRI Techniques to Evaluate Exercise Impact on the Aging Human Brain
}

\author{
Bonita L. Marks and Laurence M. Katz \\ University of North Carolina at Chapel Hill
}

USA

\section{Introduction}

The aging human brain undergoes a variety of structural and metabolic changes, often coinciding with, or leading to, cognitive decline (Bullitt et al., 2009). Over the past decade, investigators have been searching for better methods to detect, treat, and prevent cognitive decline. This has lead to the development of a plethora of pharmaceutical approaches with limited success. Identifying non-pharmaceutical approaches for the prevention/treatment of cognitive decline is paramount. Because of its non-invasiveness, neuroimaging is fast becoming a preferred technology for evaluating brain structure and function. In addition, exercise is being recognized as a potential adjunct modality for preventing or reducing structural decline in the brain and perhaps attenuating corresponding cognitive decline. These two methodologies can work in tandem: first, for identification of subtle changes in the brain not detectable via standard cognitive testing and second, for application of appropriate exercise regimes shown to be associated with healthy brain aging. Taken together, disruptions in cognitive function may be delayed, or even halted, but only if intervention occurs "soon enough". The obvious questions to answer are: 1) What is "soon enough"? 2) What type of neuroimaging might be "best"? and, 3) What kind of exercise? Simple questions with no simple answers. This chapter will begin with common, often overlooked issues regarding the use of exercise as a research modality and then progress to incorporating exercise into neuroimaging studies.

\subsection{Sedentary and unhealthy}

A sedentary lifestyle, low aerobic fitness and obesity are associated with both cardiovascular and cerebrovascular diseases (Burns et al. 2008). Research over the last decade has shown that 6 months of aerobic exercise may reduce or prevent brain volume atrophy in the prefrontal brain region related to executive function and memory in the aged (Burns et al., 2008; Colcombe et al., 2006; Erickson et al.,2009). It has also been suggested that aerobic fitness and obesity may selectively impact brain regions as well as different hemispheres (Cronk et al., 2009; Gustafson et al., 2004, 2008; Marks et al., 2007, 2010; Raji et al., 2009; Soreca et al., 2009; Ward et al., 2005). For instance, greater aerobic fitness has been moderately associated with greater cerebral white matter integrity in the anterior and middle cingulum regions on the left side of the brain whereas a higher body mass index and higher abdominal girth have been significantly associated with lower cerebral white matter integrity in the posterior cingulum region on the right side of the brain (Marks et al., 2010). 
This of course has implications beyond executive dysfunction; disruption of cerebral white matter integrity in the middle-posterior cingulum regions could impact motor movement, learning, and reading comprehension. Early transcranial doppler studies concluded that aerobic exercise may be beneficial for maintenance of cerebral blood flow (Marks et al., 2000; Orlandi and Murri, 1996). A decade later, cerebral blood vessel morphology studies suggested physically active older adults have younger-looking cerebral vasculature (Bullitt et al., 2009, 2010).

However, the retention and improvement of human brain plasticity via exercise is still not well understood. Despite animal studies demonstrating that exercise may promote neurogenesis, and human studies demonstrating a maintenance/increase in brain volume with exercise (Cotman et al., 2007; Ferris et al., 2007; van Praag et al., 1999), there is little information demonstrating the mechanism(s) for such changes. Furthermore, much of the evidence is equivocal as to whether these brain adaptations, presumably due to physical exercise, equates to improved cognitive function (Colcombe et al., 2003; Etnier and Nowell, 2006; Heyn et al., 2004; Kharti et al., 2001).

These aforementioned discrepancies may be due, at least in part, to the state of flux with research in this area. Numerous neuroimaging techniques are being used and the technology itself is rapidly changing. Cognitive tests commonly used for those with known cognitive deficits may not be sensitive enough to detect subtle cognitive changes in presumed healthy community dwelling elderly. Furthermore, researchers are using a variety of exercise paradigms, some of which are not reproducible due to lack of reporting standard exercise prescription procedures. Other factors such as age, gender, training status, and diet, known to be potential confounders in exercise and aging studies, are often overlooked. Finally, there is confusion in which term to use to simply identify the exercise paradigm itself. All of these factors make comparisons across studies difficult and the ability to draw definitive conclusions impossible (American College of Sports Medicine, 2010; Leasure and Jones, 2008; Lommatzsch et al., 2005).

Therefore, the aims of this chapter are threefold: 1) Clarify the use of exercise, physical activity and related terms as profiling variables versus intervention modalities, 2) Review neuroimaging techniques currently being used to study the impact of exercise and physical activity on the aging human brain structure, and 3) Highlight the pros and cons for use of such methods with exercise paradigms.

\section{Is it physical activity, exercise, or fitness?}

Physical activity is associated with changes in brain structure. Regular exercise improves brain function. High aerobic fitness mediates cerebral white matter integrity. Do all these statements mean the same thing, or are there subtle differences in interpretation rendering the results difficult to compare?

\subsection{Defining "exercise"}

The terms "physical activity", "exercise", and "fitness" are often used interchangeably. But as with any discipline, these terms have distinct connotations and therefore should not be use as mere synonyms. To add to the terminology confusion, a myriad of additional phrases are incorporated in an attempt to better clarify the exercise paradigm. Typical terms include, but are not limited to: aerobic fitness, health fitness, physical fitness, calisthenics, circuit training, core training, resistance training, stretching and toning, strength training, and 
weight lifting. Thus, physical activity or exercise could mean participating in a marathon or dance class, lifting a $10 \mathrm{~kg}$ medicine ball, raking leaves, meditating while performing yoga, or simply walking around a shopping mall.

\subsection{Working definitions}

Exercise scientists and physical educators continually find themselves clarifying the words that describe their work and this debate has raged for decades. For instance, the term physical activity is classically defined as any bodily movement that results in muscular contractions and increases energy expenditure above that which is used during rest (USDHHS/NHLBI, 2008). In contrast, the term exercise is defined as "the regular or repeated use of a faculty or bodily organ" (Meriam Webster Free Dictionary, 2011). Thus, the term physical activity is often used due to its broader utility, but the term exercise should be used whenever the researcher's intent is to demonstrate the impact of repeated exposure to a specific type of physical activity. Therefore, exercise can be considered a structured sub-category of physical activity, with specific dosing parameters that result in health maintenance and/or improvement (Caspersen et al., 1985). The term fitness, in biological terms, simply means the ability of an organism to survive and reproduce. This generic term is most often used to connote one's health status and is expanded as needed (i.e., health fitness, physical fitness, aerobic fitness, brain fitness). The American College of Sports Medicine (1990) suggested the following definition be used for physical fitness: "fitness is the ability to perform moderate to vigorous levels of physical activity without undue fatigue and the capability of maintaining such ability throughout life." Obviously, this exercise science-based definition can be applied to the neurological system as well, suggesting that brain fitness can be defined as the ability to perform daily cognitive tasks without undue mental fatigue or memory impairment and the capability to maintain cognitive abilities throughout life.

\section{Acute versus chronic exercise participation}

Distinctions need to be made between the acute versus chronic impact of exercise on a physiological system, in this case, the brain. While it is important to know the short-term impact exercise has on physiological systems from a biological or safe participation standpoint, the establishment of long-term health benefits attributed to exercise exposures must account for the chronic adaptations due to historical (i.e., long-term) participation in an exercise regime. It is well-established that exercise is an acute stressor, thereby resulting in (relatively) immediate elevations in blood flow, heart rate, oxygen uptake, respiration, and increased circulation/uptake of most hormones and many metabolic substrates. However, the question remains, do any of these acute exercise responses, when experienced multiple times throughout the week, over several months to many years (i.e., chronic exposure), impact the brain in such a way as to become neuro-protective and prevent or attenuate neurological degeneration and cognitive decline commonly attributed to unsuccessful brain aging?

\subsection{Cross-sectional or outcome study?}

Evaluating the brain at one point in time with a selection of a population is a cross-sectional study. One is able to infer relationships between brain structure/function and a host of variables, ranging from cognitive test scores to health fitness ratings. This is an excellent starting point and is where most of the exercise neurobiology literature is currently focused, 
likely due to time, facility limitations, and monetary constraints. However, care must be taken when reporting the results from cross-sectional studies. Regardless of the strength of the associations, results should not be reported in such a way as to infer causation. Cross-sectional research has pointed the way towards the need for more controlled, randomized longitudinal outcome studies which can take the significant associations one step further and determine causation of an intervention. While acute outcome studies are able to state how exercise stresses the brain on an immediate basis, only longitudinal outcome studies will be able to recommend more definitive exercise dosing guidelines for maintaining and/or improving brain health over a lifetime. Even then, the recommendations will likely be for specific populations, a specific gender, or specific types of physical activity. It will take several years to arrive at the more global health fitness recommendations now common in the cardiovascular literature. There is plenty of work ahead for innovative exercise-focused neuroscientists.

\section{Media releases}

The biggest blunder that has been occurring with the current brain studies is the pseudoscience reporting in the popular press. When public dollars are funding the research, it is important to get the science results out to the public in a media format that is understandable for the layman. However, the information is often unwittingly misrepresented by the media, resulting in conflicting reports when different modalities/populations are investigated, or worse, the media report leaves the impression that brain researchers are somehow privy to reading someone's mind. In the exercise neuroscience field, media interviews with researchers who are not trained in the exercise sciences or knowledgeable in the exercise design of a study has resulted in less than accurate interpretations of the study's purpose, strengths and/or weaknesses. This can have a dampening effect on future exercise neuroscience studies and may lessen the scientific integrity of the research itself. Thus it is critical that researchers understand the basics of the modality being used - in this case, exercise is the modality.

\section{The exercise dose-response}

To be comparable across studies and to better determine the most efficacious exercise plan for promoting successful brain aging, researchers and clinicians need to attend to the multifaceted nature of the exercise prescription, or dosing, components. These components can be manipulated in a variety of ways so as to not only meet the research needs but also ensure that the participants will stick with the program. While not everyone is going to love to exercise, the exercise program should be designed to accommodate one's abilities, interests, and health status. Note that the exercise prescription is individualized not as a function of age or gender per se, but rather, it is individualized as a function of personal interests and health-fitness limitations. For research, the trick is to create a general exercise prescription for an entire group while maintaining an individualistic approach, to ensure the safety of each participant, prevent drop-outs, yet still be efficacious for the research goal. That is the "art" of an exercise prescription.

\subsection{Components of an exercise prescription}

When exercise is being used as a research tool, neurobiology researchers should consider the FITT + P paradigm (frequency, intensity, time, type, progression) of an exercise prescription 
recommended by the American College of Sports Medicine (ACSM, 2009). Precisely identifying each of these components within an exercise neurobiology study makes comparison across studies, replication of results, and advancement of the exercise science of neurobiology much more accurate. Furthermore, it makes providing global recommendations to the public easy. Vaguely described exercise protocols are one of the major pitfalls encountered in the neurobiological literature utilizing exercise as a treatment modality. Often the research outcomes are either un-interpretable or non-generalizable. Manipulation of any one of the five components in the FITT $+\mathrm{P}$ paradigm can alter the intervention outcome significantly, and varying more than one component within a study must be done carefully. Ultimately the goal is to determine what type(s) of exercise recommendation(s) will best facilitate brain health maintenance. Reproducibility of the exercise prescription is paramount so that the findings can be applied across various physical activities and different populations.

\subsubsection{Frequency ("F") of exercise}

How "often" one exercises is a critical component of the exercise dose-response. It depends not only upon the health status of the individual, but also the type (or modality) of exercise. To improve cardiovascular health (or aerobic fitness), metabolic and lipid profiles, and body composition, 3 days per week is the recommended minimum number of times one should exercise (ACSM, 2009). However, if a person is at either extreme of the physical fitness continuum, (i.e., extremely deconditioned/inactive versus highly fit/active), then multiple daily sessions of very short duration (i.e. time) or nearly daily sessions of moderately long sessions may be instituted. Hence, there is a distinct relationship between frequency of exercise and duration of exercise. Simply put, the amount of time (in minutes) one should expend in a given exercise session is partially determined by how frequently one is exercising on a daily or weekly basis. Furthermore, the 3-days-per week minimum recommendation only applies to aerobic conditioning. Strength conditioning should be performed 2-3 days per week with the goal to alternate muscle groups being trained, and flexibility training recommendations is a minimum of 2 days weekly. Fitting all three of these exercise components (aerobics, strength, flexibility) into one exercise session can cause an exercise session to require at least one hour of time. Therefore, it is common to break up the exercise program into "aerobic" training days and "strength/flexibility" training days, resulting in exercising almost daily.

\subsubsection{Intensity ("I") of exercise}

How "hard" one exercises has many physiological parameters to consider including heart rate response, perception of effort, and workloads on various types of equipment. All of these factors contribute to the "intensity" of the exercise prescription and are manipulated according to the desired outcomes (Nieman, 2010).

\subsubsection{Heart rate}

If aerobic conditioning is desired, then the recommendation is for one to exercise within a "'stimulus zone". This zone is based upon one's health status and a percentage of one's agepredicted maximum heart rate $(220$ - age). For the average individual, a "moderate" intensity stimulus zone is recommended. As can be seen in Table 1, a moderate heart rate stimulus zone would be $64-76 \%$ of one's maximal predicted heart rate. So if one is 50 years old, the predicted maximal heart would be 170 , and the heart rate training stimulus zone would be 109 to $129 \mathrm{bpm}$. 


\begin{tabular}{lcccc} 
Intensity & \%HRR & \%Max HR (bpm) & RPE Range & $\%$ 1-RM \\
\hline Moderate & $40-59$ & $64-76$ & $12-14$ & $40-69$ \\
Hard / Vigorous & $60-84$ & $77-93$ & $14-15$ & $50-69$ \\
\hline
\end{tabular}

Source: Modified from: Nieman, D. Exercise Testing and Prescription, A Health Related Approach. $7^{\text {th }}$ Edition, New York: McGraw Hill Publishers, 2011, pp 180, Table 6.3, Classification of Physical Activity Intensity

Table 1. Intensity scales equating verbal descriptions to percent heart rate reserve (\%HRR), percent heart rate max (\%HRmax), rating of perceived exertion (RPE) based on the Borg 6-20 scale, and percent of a one-repetition maximum (\%1-RM) strength test.

A slightly more complex, but more accurate way to prescribe aerobic exercise intensity is by using the Karvonen formula, a mathematical formula using percentage of one's heart rate reserve (maximal heart rate - resting heart rate). This requires knowing one's maximal heart rate (or estimating it as shown above), knowing one's resting heart rate (being able to take one's pulse rate at rest) and using the percentages listed in Table 1. Because this calculation more closely represents oxygen consumption requirements, the percentages shown in the table are slightly lower than the ones used with the age-predicted heart rate max method just described. Thus, the formula for determining a moderate-intensity heart rate stimulus zone using the Karvonen Method is as follows:

$$
\begin{aligned}
& \text { [(Maximal Heart Rate - Resting Heart Rage }) * 40 \% \text { ] + Resting Heart Rate } \\
& \text { [(Maximal Heart Rate - Resting Heart Rage }) * 59 \% \text { ] + Resting Heart Rate }
\end{aligned}
$$

Thus, if our 50-year old person had a resting pulse rate of $75 \mathrm{bpm}$, using the Karvonen method to determine his exercise stimulus zone, his heart rate training stimulus zone would be 113 to $131 \mathrm{bpm}$.

\subsubsection{Perception of effort}

Sometimes heart rate responses are modified by medications or the exercise participant simply cannot take his/her pulse rate. In that case, exercise can be prescribed based on one's perception of the exercise intensity. This is called "rating of perceived exertion", or RPE. The most common RPE scale used is Borg's 6-20 scale, which at moderate intensity exercise, correlates well with the heart rate response. For instance if a person rates his level of exertion to be between 12-14, the heart rate is generally within 120-140 beats per minute. It does take about 3 practice sessions for the user to become familiar and comfortable with this scale in order to get the most accurate RPE scores (Borg, 1985). For a more complete understanding of using perceived exertion, an excellent applied book is "Perceived Exertion for Practioners" (R.J. Robertson, 2004, Human Kinetics Publishers).

\subsubsection{Workload}

When utilizing equipment for exercise training, the intensity of training will in part be mediated by the workload setting employed. For instance, if a moderate intensity is desired for lifting weights on a machine, a percentage of what a person is able to lift maximally one time (\% 1-RM) maybe used. As seen in Table 1, to strength train at a moderate intensity, approximately $40-69 \%$ of a 1-RM will be recommended. That means, if the maximum weight one is able to lift is 100 pounds, then the training weight stack should be between 40 and 69 pounds (or, $45 \mathrm{~kg} \max =18$ to $31 \mathrm{~kg}$ ). If using a treadmill, the exerciser would need 
instructions as to how to set the speed and percent grade; if using a cycle ergometer, the exerciser would need to know how to set the resistance and at what speed to pedal. Sometimes this is determined by an entry exercise test and the settings are based upon a percentage of their max test results; other times it is arbitrarily determined and governed simply by determining a "comfortable" pace in order to attain a desired heart rate or RPE range. Using the latter method will enable the researcher to permit the exerciser to exercise on a greater variety of equipment, thereby helping to reduce exercise boredom and dropping out of a study. However, a word of caution: if the goal of the research is to determine the impact of a certain TYPE of exercise on the brain over a certain period of time, then the researcher must give explicit instructions as to which equipment use is permissible for exercise research participation. Sometimes, giving a research volunteer too many choices can truly confound interpretation of the research results. Thus, while that new exercise club down the road may be convenient and affordable for the study, the researcher must determine how precisely the exercise prescription must be adhered to and consider the consequences if a subject veers off course.

\subsubsection{Time (“T"), or duration, of exercise}

The first "T" of the FITT + P paradigm is Time. How "long" one exercises, or how much time is required to achieve a desired fitness benefit, depends upon one's health status and/or fitness goals, and as stated above, the frequency of exercise. If one is very deconditioned, then multiple sessions of brief duration may be recommended. These brief durations may be as little as 5 minutes. It is common for those with a fragile health status or simply deconditioned due to inactivity (but otherwise considered healthy) to be given an intermittent exercise prescription consisting of 5 minutes of physical activity interspersed with an equal amount of rest, with that dose repeated twice more in succession so that an equivalent of 15 minutes can be accrued. As one successfully adapts to the exercise stimulus, the rest sessions will be reduced so that eventually the previously deconditioned person can exercise for 15 continuous minutes. Once a baseline level of aerobic endurance is attained, then strength conditioning can be safely and effectively added to the exercise program. As indicated above, an exercise session focusing only on aerobic conditioning can require 15 to $30+$ minutes. A strength conditioning program may also require $30+$ minutes if the entire body is to be trained in one session. Flexibility training can be a stand-alone program or be incorporated into the regular exercise program as part of a warm-up and/or cool-down routine. Thus, flexibility training can take as little as 5 minutes or as long as 30 minutes, depending upon the nature of the training.

While any one component of an exercise program may eventually take about $30+$ minutes, it is standard to also incorporate a brief 5-10 minute warm-up before entering the "stimulus zone" and a 5-10 minute cool-down after completing the "stimulus zone" work-out. The warm-up is usually a lighter version of the stimulus and is to ensure the body is prepared to be stressed, whereas the cool-down is usually a relaxing set of stretches to enable the body to return to the pre-exercise non-stressed state. Therefore, at least 30 minutes needs to be allotted for the first week of a beginning exercise session (5 minute warm-up, 20 minute stimulus, 5 minute cool-down), and more time thereafter as one's exercise prescription is upgraded, or progressed through several weeks of a research study.

Another aspect of "time" is the actual timing of the exercise - that is, time of day. While this does not impact the dose-response of exercise per se, it does impact the effectiveness of the 
exercise plan if the time of day allotted to exercise is not compatible with the exerciser's lifestyle. For instance, if exercise is to take place under supervised conditions at a facility, the hours must be agreeable with the exercise's life - are there times available before or after one's work day, or at lunch? If recruiting a person with child care responsibilities, are there childcare services? Are weekend hours available? Other concerns are parking, commuting time, or easy bus/rail access. Will the research study pay for on-site childcare or parking?

\subsubsection{TYPE ("T") of exercise}

The other " $\mathrm{T}$ " component of the FITT + P paradigm is the TYPE of exercise (or activity) needed to achieve the stated research goals. The exercise prescription type is subdivided into three broad categories: aerobic endurance (or fitness), muscular strength/endurance, and flexibility. Of course each of these broad activity categories has numerous subtypes, thus it is crucial to specifically describe the type of activity one is to engage in. For instance, an aerobic activity is any activity that a person can complete continuously for 15 minutes or more that utilizes a large portion of the body's musculature in a rhythmic fashion. This includes common individual activities such as walking, running, swimming and cycling but it can also include games, sports and various types of dance. Muscular strength/endurance training also has many sub-types. It can consist of the traditional lifting of weights (aka weight training) or it can be termed resistance training or core training and involve not only dumbbells, free weights, or machines, but also medicine balls, resistance bands and tubing, kettle balls and one's own weight (e.g. push-ups, sit-ups). Other types of musculoskeletal training can include balance training, plyometric training, neuromuscular facilitation training, yoga, and tai chi. Flexibility training can involve static, ballistic, or dynamic stretching. Often times, strength and conditioning programs are simply called "stretching and toning", which really provides no concrete idea of the type of training actually provided. Thus, with all these options available to the researcher, creating a reproducible exercise program to investigate a particular health parameter becomes an art form. Obviously it is not possible to investigate every aspect of exercise within one study, so the researcher must narrow his/her focus to a select few options and describe them wellenough for the reader to be able to replicate. Ultimately, with enough well-designed neurobiology exercise studies, general recommendations for cerebral health will be able to be created, similar to those that now exist for cardiovascular health.

\subsubsection{Progression ("P") of exercise}

There are a variety of ways to "progress" an exercise prescription so that it remains challenging yet doable for the participant and prevents boredom or staleness. The progression of exercise is increased over the ensuing weeks at a percentage that is both safe and effective for that particular individual. The eventual goal is for one to attain a minimum of 30 or more minutes of continuous exercise on most days of the week (Haskell et al., 2007). One rule of thumb has been to increase any given exercise dose by as little as $2 \%$ or as much as $30 \%$ weekly or every other week. Another practice is to increase the duration of exercise by approximately 5 to 10 minutes every week, which might translate to a $15 \%$ increase in time week to week. If there is little room for adding additional time to an exercise session, then an extra day of training can be added on. If neither time nor frequency is an option to increase, then intensity becomes the progression target. When a person's perception of effort decreases along with lower heart rate responses with any given exercise stimulus, it is time to increase the exercise intensity. The goal is to make sure a slight overload is placed upon 
the physiological systems so that the body can continually respond and successfully adapt to the overload. Unsuccessful adaption to an overload will result in undue fatigue, unnecessary muscle soreness, and if extreme, illness and/or injury.

Perhaps the most important concept to understand is the complex interaction between intensity, frequency and duration of the exercise prescription and how manipulation of any one of these variable impacts the exercise progression and adaption. The way to avoid unsuccessful overloading is to increase only one exercise prescription component in any given exercise session. For example, if the frequency of exercise training is scheduled to be increased from 3 days a week to 4 days a week, then the duration (total time) and intensity of the exercise session should remain the same as the previous training session. If on the other hand, the intensity of exercise needs to be increased, then the duration of the exercise session should either remain the same or be decreased slightly to accommodate for the increased effort required. On the following day, the duration can be returned to its previous level as long as the "new" exercise intensity remains the same. Figure 1 outlines the basic components of the exercise prescription and can serve as a quick-reference exercise dosing

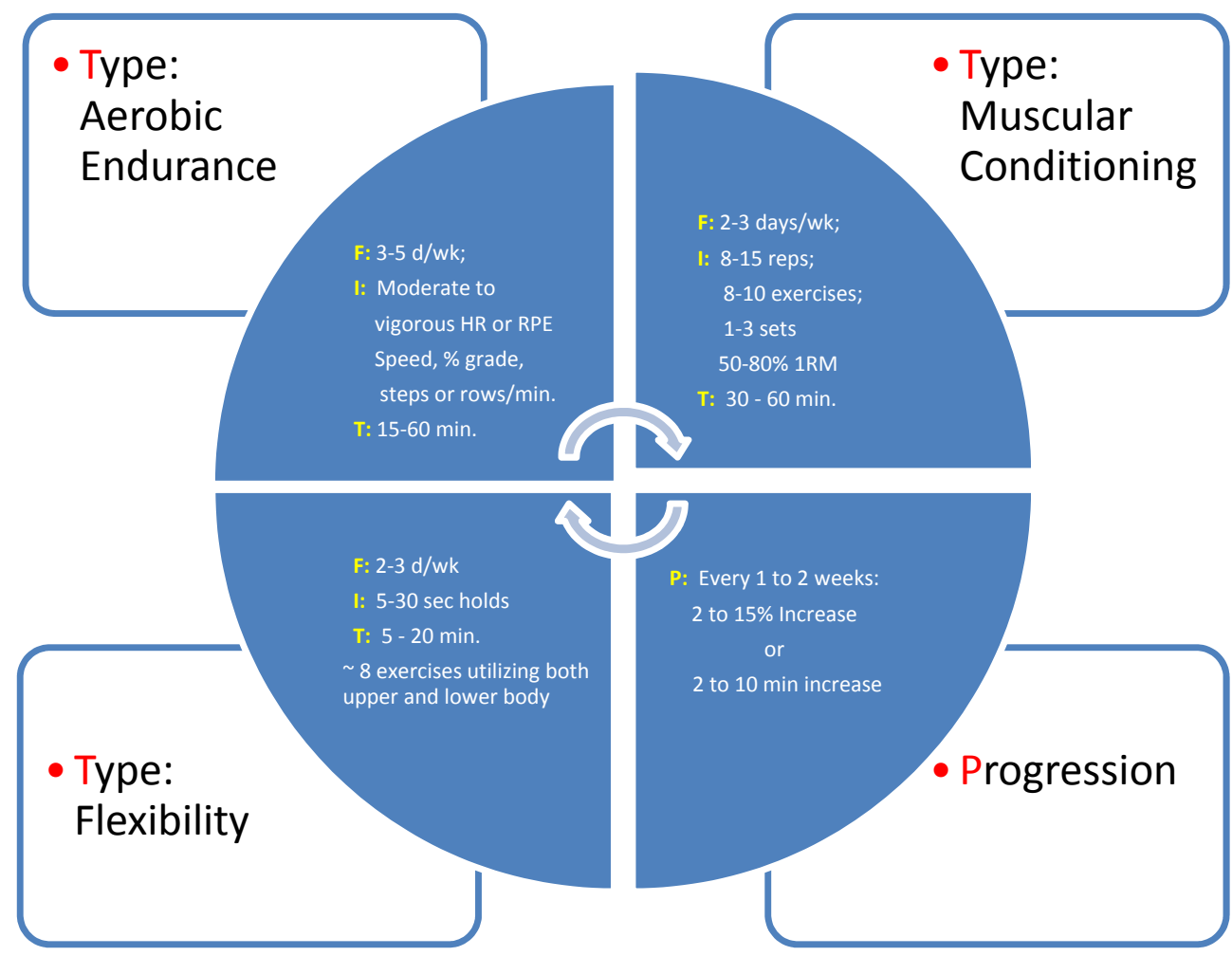

Fig. 1. Exercise prescription components featuring the FITT + P paradigm. Less active, unfit individuals would have exercise dosing at the lower ranges where as more active, higher fit individuals would have exercise dosing at the higher ranges. $\mathrm{HR}=$ heart rate; $\mathrm{RPE}=$ rating of perceived exertion. 
guide. More complete exercise prescription information is available in the ACSM's Guidelines for Exercise Testing and Prescription book (2009), updated every four years. The best advice for researchers using exercise as a research treatment arm, or clinicians using exercise as a therapeutic agent is to make sure one or more ACSM-certified exercise physiologists are a part of your team.

\section{Structural neuroimaging and exercise impact}

What type of neuroimaging is best when trying to determine how exercise impacts the [aging] brain? That depends on the question(s) one needs to answer. If the goal is to determine the long-term impact of exercise on brain structure, then structural MRIs are appropriate. Structural imaging can track changes over time due to a stimulus such as exercise. Structural imaging can involve gray and white matter volume determination ("quantity"), cerebral white matter integrity ("quality") using diffusion tensor imaging (DTI), or both. It can also involve investigating the cerebral blood vessels utilizing magnetic resonance angiography (MRA).

\subsection{Voxel-wise or ROI approach?}

The MRI analysis can take a voxel-wise approach if the researchers have no particular hypotheses regarding expected areas of impact, or the MRI analysis can take a regions of interest (ROI) approach if there are research-driven hypothesis regarding expected areas of impact from physiological knowledge. There are pros and cons to both methods. It is argued that the voxel-wise approach is less biased, less time consuming, and therefore less costly. The ROI approach is argued to be driven by scientific knowledge of the physiology, more targeted and less "look-see" exploratory type research. While the ROI approach can be more costly due to the labor-intensive outlining of the specific ROI, and costly if ROI mapping is being done by more than one investigator for reliability purposes, if automated ROI templates are available, the cost and time decrease substantially. Unfortunately, not all ROI have templates. The "con" to template mapping is that a small degree of accuracy is sacrificed due to assuming a "standard shape" for a brain region when in fact there is no true standard shape (Scheibel, 2009). Thanks to the NIH Neuroscience Roadmap Initiative, there are a variety of free resources, including software mapping templates, available on the internet to download. One such site is called MIDAS and offers brain images as well as toolkits: http://www.insight-journal.org/midas/gallery/?flash=true

\subsection{How long does it take for exercise to alter brain structure?}

While rate of decline in brain mass with aging is highly individualized, it is often stated that the "normal" brain gradually decreases in size 10-15\% with aging, and this shrinkage becomes particularly evident in octogenarians (Scheibel, 2004). The average rate of brain atrophy is between 0.9 to $1.5 \%$ per year after age 52 , with the steepest rate of decline occurring in the frontal region with concomitant cognitive decline (Dennis \& Cabeza, 2008). While it is not currently known precisely how much time is needed to facilitate structural changes (i.e., improvements) in the brain due to exercise, it is reasonable to hypothesize that relatively short-term structural change may be possible. In two separate studies demonstrating brain plasticity, as little as 1.5 to 3 months of cognitive training resulted in cortical changes in younger populations (Driemeyer et al., 2008; Haier et al., 2009). As for exercise, six months of aerobic exercise using moderate intensity walking 3 days per week 
for an hour each session not only prevented brain volume atrophy but resulted in brain volume improvement in older adults (Colcombe et al., 2006). Using voxel-based morphology, improvements in brain volume were noted in both the gray and white matter regions associated with executive function, long term memory, and general intelligence (i.e., the prefrontal and temporal cortices). These improvements were cautiously reported in terms of brain atrophy risk reduction in comparison to a stretching/toning control group such that a $16 \%$ improvement in aerobic fitness resulted in a 27 to $42 \%$ risk reduction of brain atrophy. The greatest risk reduction was in the anterior cingulate cortex. The stretching/toning group experienced a non-significant $5 \%$ increase in aerobic fitness but no volumetric information was reported for them. Although it is not known if the 5\% improvement in aerobic fitness also resulted in some volumetric improvement, it might be surmised that embarking upon a moderate-intensity aerobic exercise program which produces at least a $1 \%$ increase in aerobic fitness may attenuate aging-related brain atrophy. This was one of the first longitudinal outcome studies reporting the impact of aerobic versus musculoskeletal-type exercise on the aging brain.

Currently, little neuroimaging information is available on other modes or durations of exercise training; nor is there information regarding how quickly the human brain structure detrains. But if the brain/cerebrovasculature mirrors the heart/cardiovasculature in exercise adaptations, then like the cardiovascular system, the cerebrovascular system may lose that $11 \%$ gain in as little as 3 weeks of no training (Coyle et al., 1984). Thus, the protective effect against brain atrophy may be lost in one short month if one is unable to exercise sufficiently. An intriguing question remains, can cognitive brain training (e.g. suduko, puzzles, playing chess, Wii-games) supplant physical activity during periods of physical inactivity in order to maintain brain structure and function?

\subsection{Exercise neuroimaging study shortcomings}

Being a pioneer in exercise and aging neuroscience research also means there will likely be design flaws in the research. For instance, in Colcombe et al's study (2006), the age range was wide, 60 to 79 years with a mean age of 66 years. The study age range spanned two decades with three standard aging cohorts: the older end of middle-aged (45-64 years old), the young-old (65-74 years old), and the younger end of old (75-84 years old). No mention was made regarding how many subjects fell within each of these age cohorts, therefore it is not known if these age cohorts responded differently to the exercise programs. With no variance measure or age range provided per group on any variable, it is difficult to assume the study did not have a few inadvertent biases. A potential younger-age bias may have preexisted in the aerobic treatment group (the treatment group was on average 1.4 years younger). The stretching/toning control group had a slightly higher percentage of females ( $4 \%$ more), creating a potential gender bias. Further, the actual pre-aerobic fitness distribution per grouping was unclear. Although the mean aerobic fitness $\left(\mathrm{VO}_{2}\right)$ values were not significantly different between groups ( $23 \mathrm{ml} / \mathrm{kg} / \mathrm{min})$, the pre-intervention $\mathrm{VO}_{2}$ values ranged from 12.9 to $49.9 \mathrm{ml} / \mathrm{kg} / \mathrm{min}$. Thus there were some older individuals with pre-intervention $\mathrm{VO}_{2}$ values who would be considered highly fit and therefore have less room for improvement from any type of intervention. It is not known if an attempt was made to balance the placement of these higher-fit individuals into the two groups since the methods claim group assignment was totally randomized. Furthermore, it was reported that the aerobic group was previously sedentary, however older adults with $\mathrm{VO}_{2}$ 's exceeding $40 \mathrm{ml} / \mathrm{kg} / \mathrm{min}$ are not likely habitually sedentary. Individually, the between group differences highlighted here are small and were 
reported to be non-significant, but considered collectively, these small biases could contribute to confounding the interpretation of the results.

Sociological studies have shown that women outlive men by 4 to 10 years, thereby partially explaining why there are usually more women in research studies involving older adults. Although women tend to live about a decade longer than men, they also experience an accelerated pace of physiological decline between their seventh and eighth decade of life. Older men tend to weigh more, be more physically active and have a higher degree of aerobic fitness than older women of the same age (Spriduso et al. 2005) and women's brain volumes are smaller than men (Allen et al., 2003). Thus care must be taken when studying variables with inherent age or gender differences. Colcombe et al. (2006) made no mention of controlling for age or gender in the statistical analyses of their data in order to determine if improvements attributed to aerobic fitness change was independent of age or gender influences. It is well known that both age and gender can impact brain volumes and cognition independently (Madden et al, 2009). Failure to control for these variables can lead to potentially erroneous conclusions. For example, Marks et al. (2010) initially noted moderate positive relationships in the anterior cingulum segment between cerebral white matter integrity and aerobic fitness as measured by diffusion tensor imaging (DTI). However, upon controlling for both age and gender, only the middle and posterior cingulum segments remained significantly related to aerobic fitness. Similarly, this pattern of reduced significance was repeated in a voxel-wise brain analysis on the same data (Liu et al. 2009). Thus it is critical to control for factors that are known to impact the brain and/or aerobic fitness parameters. Lastly, neither the exercise test protocol nor the "stretching and toning" prescription was ever fully described by Colcombe et al. (2006). This lack of information makes it difficult to determine the validity of the aerobic fitness and strength training outcomes, and it is even more difficult to impart health recommendations with confidence. Hence the encouraging conclusions regarding aerobic fitness and brain improvement from this study by Colcombe et al. (2006) must be viewed with cautious optimism. There are currently a few new NIH-funded exercise trials involving both healthy and diseased older adults in progress (http://projectreporter.nih.gov/reporter.cfm), with intervention timelines and exercise protocols seemingly mirroring Colcombe et al. 's initial study (2006) . Hopefully, these newer studies will not only control for potential confounding variables but also provide sufficient exercise testing and training details to render their studies replicable.

\subsection{Magnetic resonance angiography and exercise impact}

Magnetic resonance angiography (MRA) in conjunction with DTI is helpful in determining the status of one's cerebral blood vessels. Using a process known as arterial spin labeling (ASL), the quality and quantity of the cerebral blood flow can be determined with or without perfusion. It is believed that the progressive reduction in cerebral blood flow attributed to the aging process may be caused by a reduced metabolic demand due to a reduction in neurotransmitter synthesis (Orlandi \& Murri, 1996) and/or underlying microvascular disease (Bullitt et al., 2010). The consequential neural atrophy results in smaller cerebral arteries, increased intracranial resistance and slower arteriole vasomotor reactivity (Orlandi \& Murri, 1996). Even though studies suggested aging may be associated with smaller cerebral vessels, Bullitt et al. (2010) reported that vessel diameter reductions may be compensated for by an increase in vessel number and that both larger and smaller vessels were impacted. In a sub-study comparing active versus inactive older adults, Bullitt et al. (2009) reported significantly lower vessel tortuosity along with a higher number of 
smaller vessels. In a separate conference paper, although cerebral blood flow velocity did not change, Rahman et al. (2008) reported less variance in the cerebral blood flow velocity in those with higher physical activity levels. To examine both the cerebral vasculature as well as cerebral blood flow, arterial-spin labeling (ASL) would be required. For either the blood oxygen level-dependent effect (BOLD) or ASL methods, intravenously (IV) injected contrast agents will produce more distinct images. However decent (but not great) images can be obtained without the IV injections. Not using invasive procedures is certainly more appealing to the volunteer subject and helps to contain the imaging costs as well.

\section{Functional neuroimaging and exercise}

If one is interested in determining which regions of the brain are being activated /oxygenated during an exercise or cognitve task, a functional MRI (fMRI) using the BOLD response would be needed. For exercise studies, the obvious hurdle to overcome is movement as most movement causes disruption in the scanning process and poor images are created. Whereas cognitive psychology has forged numerous research pathways using fMRI with BOLD contrasts to determine regions of activation in the brain during various cognitive tasks, this has not been the case with exercise training interventions. Clearly there is a need for this type of research if one desires to investigate changes in cerebral blood flow or neural hormonal factors due to an exercise stimulus from either an acute exercise bout or in response to a chronic adaptation. The stumbling block to overcome is the exercise test itself. Most exercise studies use upright testing protocols on equipment that are large and bulky with both metal and electronic parts. All of this precludes testing within the scanning room due to the magnetic field. Furthermore, by the time the subject could be transferred from the exercise apparatus to the scanning bed, critical time would be lost such that the exercise impact on the cerebrovascular system would likely be missed in all but the most deconditioned subjects.

\subsection{MRI-friendly leg cycle ergometer}

Therefore, up to this point, the more feasible methodology for cerebral blood flow investigations with exercise have been with using transcranial dopplers (TCD) and/or electroencephalography (EEG). Although these methods also have difficulty with accurate measures during movement, they are in comparison, lower in cost and easier to administer than an fMRI study. However, for approximately \$75,000 (US\$), the Lode MRI-compatible recumbent leg cycle ergometry system can now be purchased from ELECTRAMED Corporation, located in Flint, Michigan, http://www.electramed.com/MRI\%20ERGOMETER \%20CARDIOLOGY\%20_Details.htm. This would enable the researcher to conduct exercise tests while the subject remains in the MRI unit. Also available are MRI-compatible electrocardiography and blood pressure measurement units, thereby solving the equipment issue. Unfortunately, this particular equipment model is only compatible with a $1.5 \mathrm{~T}$ MRI scanner and only with select manufacturers. Given that most research is now being done on $3.0 \mathrm{~T}$ or $4.0 \mathrm{~T}$ scanners, this ergometer may not be useable for many research protocols. The final issue left to resolve is an acceptable exercise protocol that would be taxing enough yet involve minimal movement from the torso up during scanning sequences. One potential resolution would be to develop an intermittent exercise test protocol so that exercise bouts would take place during the imaging sequence changes, akin to an event-related design. Since stimuli in an event-related design are presented as isolated events of short duration, a brief 
cycling set that progressed in intensity with each event presentation could be incorporated (Carter and Sheih, 2010). Obviously, much pilot work would need to be done to determine the exact power outputs required to elicit measureable BOLD signals.

\subsection{Exercise mental imagery}

If actual physical exercise testing is not possible, there is still one other avenue to determine cerebral activation during an exercise task: mental imagery. For example, a sport psychology study investigated motor imagery of the golf swing to determine brain region activation. Using the sensori-motor homunculus map as a guide, Ross et al. (2003) compared the amount of fMRI BOLD response in brain regions related to the golf swing between novice versus expert golfers. It was determined that the greater the golf handicap, the greater the region of activation (greater than $2 \%$ ) in specific somatatropic regions of interest relevant to golf. The powerpoint presentation can be downloaded from the internet with a search engine. A very recent BOLD fMRI study (Cremers et al., 2011) investigated mental imagery consisting of subjects envisioning themselves either walking, standing, or lying down (block design). Their imagined walking (speed $=2.3 \pm 0.4 \mathrm{~m} / \mathrm{s}$ ) was associated with activation in the right dorsolateral prefrontal cortex, posterior parietal lobule, and the left cerebellar hemisphere. Therefore, it might be interesting to conduct an imagery intervention study to determine the acute response to an imagined exercise stress test as well as an imagined chronic response to a long-term exercise intervention. Studies of this nature have not yet been reported.

\section{Testing pearls and pitfalls}

Neuroimaging studies are expensive. Exercise testing and training are expensive. Recruitment drop-outs are expensive. And botched tests are expensive. They are expensive in terms of time, money and patience. Neuroimaging and exercise testing aged individuals bring a unique set of challenges to intervention research. There are the standard safety issues to consider when using a neuroimaging technique or conducting a physical exercise test; but the less obvious issues of comfort and trust sometimes slip by unasked, until it is too late and the subject has dropped out of the study. Therefore, when screening an older individual for an imaging and/or an exercise study, the following question must be asked: can the volunteer complete the testing protocol accurately and in relative comfort? The researcher must ascertain that the older volunteer can hear, see, follow directions, and adhere to the instructions. Volunteers must be able to complete enough of the exercise protocol to get valid physiological baseline data and/or remain motionless and pain free in the MRI scanner anywhere from 15 to 120 minutes. The brief breaks afforded between imaging sequences when a subject is free to move slightly may be insufficient. Arthritis, nasal-sinus drainage, and circulatory issues have thwarted many research MRI scans. For a first-time MRI scan, volunteers may back out at the last minute due to unanticipated fright (hence a simulator is an invaluable resource) or the irrational worry that the MRI will read their minds (thanks to outlandish media stories). Thus, the researcher must design protocols with both the science and the targeted subject population in mind.

\subsection{Exercise testing versus physical activity recalls}

Actual measurement of aerobic fitness, as opposed to estimating it in some fashion, is usually preferable. There are a variety of reference books available detailing exercise protocols for various health and fitness statuses. The researcher who wants to include 
exercise in the research design should obtain the ACSM Guidelines for Exercise Testing and Training (2009). A good textbook is Nieman's (2010) exercise prescription textbook used for training undergraduates in exercise science. However, there are times when it is inconvenient, illogical, or cost-prohibitive to conduct a fitness test. For those times when actual exercise testing is ruled out, there is a rather good non-exercise aerobic fitness estimation equation that is quite easy to use, providing one is trained in obtaining a valid physical activity recall. While it can be difficult to get accurate physical activity recalls beyond a few weeks, a seasoned investigator in physical activity recall questionnaires can elicit excellent responses, even recalls spanning several years.

One physical activity recall formula for estimating aerobic fitness has been in the literature since 1990. It was gathering dust until recently when we used it for a retrospective analysis exploring the role aerobic fitness might have on cerebral white matter integrity on both younger and older adults (Marks et al., 2007). Ever since that publication, we have been getting inquiries about the formula and how to use it. The formula was developed and tested at the Cooper Aerobic Institute in Texas on over 2,000 U.S. Air Force personnel ranging in age from 18 to 70 years. The subject population included males and females, fit and unfit, healthy and unhealthy. The estimated aerobic fitness value $\left(\mathrm{VO}_{2}\right)$ has a standard error of about $5 \mathrm{ml} / \mathrm{kg} / \mathrm{min}$. This formula is very good for cross-sectional, population-based studies when the purpose is to simply categorize one's fitness level. However, the error range is a bit too high and the fitness categorizing a bit too vague for pre-post research designs where $\mathrm{VO}_{2}$ change is a critical factor. For that, $\mathrm{VO}_{2}$ does need to actually be measured. Although the formula tends to underestimate the highly fit and over-estimate the very low fit, all subjects are still able to be categorized accurately into a fitness level (e.g., low fit, average fit, high fit). The estimation formula and its accompanying physical activity rating scale (PARS) are contained in Table 2 and Table 3 below:

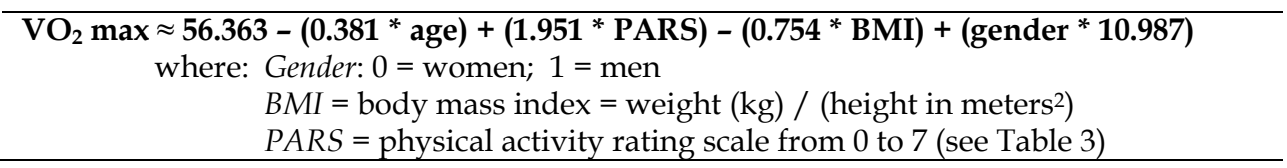

Table 2. Estimated Aerobic Fitness ( $\mathrm{VO}_{2}$ max) (Jackson et al., 1990).

\section{Limitations and suggestions}

The good news is, lines of inquiry utilizing neuroimaging are still rather novel and as such, there is much to study. The bad news is, these lines of inquiry utilizing neuroimaging are still rather novel and there is much to learn, so omissions and/or mistakes in research design are to be expected. Investigating how exercise impacts the brain is akin to the first studies investigating how exercise impacted the heart and its related vasculature several decades ago.

A limitation in several structural imaging studies (e.g., Marks et al. 2010; Bullitt et al. 2009; Colcombe et al., 2006) was lack of cognitive function testing - it is unknown if the improved brain structures found in the more active subjects would have translated into better cognitive function. Adding cognitive testing with magnetic resonance imaging (MRI) may help detect subtle changes that the standard cognitive test batteries if used alone, cannot. If the MRI is able to detect changes, independent of the cognitive tests, a therapeutic program could be implemented at an earlier stage of decline and perhaps be more effective in reducing further impairment. 
Directions: Query the participant regarding his/her extent of physical activity using the activity descriptors below as well as established metabolic tables for physical activity.

A. No regular participation in programmed recreational sport or physical activity:

$0=$ avoid walking or exertion (always use elevator, drive whenever possible instead of walking.)

1 = pleasure slow walking, routinely use stairs, occasionally heavy breathing or perspiration

B. Regular participation modest/moderate physical activity ( e.g. golf, horseback riding, calisthenics, gymnastics, table tennis, bowling, weight lifting, yard work etc):

$2=10$ to 60 minutes per week

$3=$ over one hour per week

C. Regular participation in heavy physical exercise (e.g. jogging, running, swimming, cycling, rowing, skipping rope, running in place, tennis, basketball, or handball etc.):

$4=$ run less than 1 mile per week or spend less than 30 minutes per week in comparable heavy physical activity

$5=$ run 1 to 5 miles per week or spend 30 to 60 minutes per week in comparable heavy physical activity

$6=$ run 5 to 10 miles per week or spend 1 to 3 hours per week in comparable heavy physical activity

7 = run over 10 miles per week or spend over 3 hours per week in comparable heavy physical activity

Table 3. Physical Activity Rating Scale (PARS; Baumgartner and Jackson, 1995).

There are also scanner issues to deal with when a study design goes from a single acute scan to repeated scans over several months or years. Your scanner must be intra-reliable (i.e, a measure today will yield relatively the same results tomorrow). It is well known among neuroimaging technicians that scanners "drift" over time, therefore it is important to keep track of the drift so you can be sure changes that are seen months from the initial scan are corrected for the drift. Along these same lines, in order to get larger sample sizes, multiple sites may be needed. Therefore all the scanners used must be determined to be inter-reliable. This is generally accomplished with phantom testing.

It is equally important to account for individual brain plasticity - the investigator must understand the normal brain changes over time independent of any treatment so that intervention changes seen can be distinguished from random occurrence or normal aging.

Lastly, dehydration is an issue that only recently has begun to be accounted for in neuroimaging research studies. Care must be taken to ensure the research volunteers are euhydrated, otherwise, the question that may arise when a brain volume increase is reported: Is the increase in brain volume "true"? Or is it due to dehydration known to plague not only the elderly but also exercisers who exercise in a hot environment and may not have hydrated sufficiently? A simple way to account for hydration status is to obtain a urine sample and test it for urine specific gravity using either a dip stick (aka chem. strip) or a small handheld refractometer. A non-smelly light straw-colored urine would suggest the person was adequately hydrated. If a more precise objective measure is needed, the urine specific gravity reading should be between 1.010 to 1.020 (Armstrong et al., 1998). 


\section{Conclusions}

Aerobic fitness not only facilitates improved oxygen delivery and utilization in the cardiocerebral vascular systems, improved oxidative capacity has been shown to up-regulate expression of important neuronal growth factors such as insulin-like growth factor I (IGF-I), brain-derived neurotrophic factor (BDNF) and related protein precursors in animal models (Ding et al., 2006; van Praag et al., 2005). Furthermore, aerobic fitness may mediate improved cerebral white matter integrity via the intricate adaptations that take place on the neural-humoral level during exercise (Marks et al., 2010). Therefore, exercise outcome studies trials need to include not only structural imaging detailed in this paper, but also hormonal measurements and perfusion imaging.

In summary, the research questions that remain to be answered are: What is a sufficient exercise dose for the brain? How much, or how little, exercise is really needed to maintain brain structure and cognitive function? Will any type of physical activity do? Will the resultant health recommendations for the brain be complementary to the current guidelines for cardiovascular health?

To move the future of brain training research forward, we must continue to revisit the past ground-breaking cardiovascular research studies and modify them for the brain. Ancient physicians and philosophers like Hippocrates and Cicero espoused the benefits of exercising both the body and the mind, and here we are, 2,000 years later, scientifically documenting the neurobiological benefits of exercise. Several investigators have been using a few standard cardiovascular disease risk reduction guidelines with success in maintaining brain volume in older adults, but so many more exercise options remain to be explored. Hopefully it will not take another 50 years to firmly establish exercise guidelines for maintaining and enhancing brain health with exercise.

\section{Acknowledgment}

The University of North Carolina at Chapel Hill's Libraries provided support for open access publication.

\section{References}

Allen, J.S., Damasio, H., Grabowskia, T.J., Brussa, J. and Zhang, W. (2003). Sexual dimorphism and asymmetries in the gray-white composition of the human cerebrum, NeuroImage, 18:880-894

American College of Sports Medicine. (2009). ACSM's Guidelines for Exercise Testing and Prescription, 8th Edition, New York: Lippincott, Williams and Wilkins

American College of Sports Medicine. (1990). The recommended quantity and quality of exercise for developing and maintaining cardiorespiratory and muscular fitness in healthy adults. Med Sci Sports Exerc, 22:265-274

Armstrong, L.E., Soto, J.A., Hacker, F.T., Casa, D.J., Kavouras, S.A., Maresh, C.M. (1998). Urinary indices during dehydration, exercise, and rehydration. Int. J. Sport Nutr. 8: 345-355

Borg GAV. (1985). An introduction to Borg's RPE Scale. Ithaca, NY:Movement Publications.

Baumgartner, T.A. \& Jackson, A.S. (1995). Measurement for Evaluation in Physical Education and Exercise Science, (p. 289) Brown \& Benchmark Publ., Madison:WI 
Bullitt E, Zeng D, Ghosh A, Aylward SR, E, Lin W, Marks BL, Smith K. (2010). The effects of healthy aging on intracranial blood vessels visualized by magnetic resonance angiography. Neurobiology of Aging, 31:290-300

Bullitt E, FN Rahman, JK Smith, E Kim, D Zeng, Katz LM, Marks, BL. (2009). The effect of exercise on the cerebral vasculature of healthy aged subjects as visualized by magnetic resonance angiography. Am J Neuroradiol, 30:1857-1863

Burns JM, Cronk BB, Anderson HS et al. (2008). Cardiorespiratory fitness and brain atrophy in early Alzheimer's disease. Neurology, 71:210-216

Caspersen CJ Powell KE, Christenson GM. (1985). Physical activity, exercise, and physical fitness: Definitions and distinctions for health-related research. Public Health Reports, 100:120-131

Colcombe SJ, Kramer AF. (2003). Fitness effects on the cognitive function of older adults: a meta-analytic study. Psychol Sci, 14:125-130

Colcombe SJ, Erickson KI, Scalf PE et al. (2006). Aerobic exercise training increases brain volume in aging humans. J Gerontol-A Biol Sci Med Sci, 61:1166-70

Cotman CW, Berchtold C, Christie L. (2007). Exercise builds brain health: key roles of growth factor cascades and inflammation. Trends in Neuroscience, 39:464-472

Coyle EF, Martin WH, Sinacore DR, Joyner MJ, Hagberg IM, Holloszy JO. (1984). Time course of loss of adapatations after stopping prolonged intense endurance training. J Applied Physiology: Respiratory, Environmental and Exercise Physiology, 57:1857-64

Crémers J, Dessoullières A, Garraux G (2011). Hemispheric specialization during mental imagery of brisk walking. Hum Brain Mapp, Mar 21. doi: 10.1002/hbm.21255. [Epub ahead of print] Last Accessed April 25, 2011

Cronk BB, Johnson DK, Burns JM. (2009). Body mass index and cognitive decline in mild cognitive impairment. Alz Dis Assoc Dis., Epub Ahead of Print: 2009 June 30. PMED:19571736

Dennis NA \& Cabeza R (2008). Neuroimaging of Healthy Cognitive Aging. In: The Handbook of Aging and Cognition, $3^{\text {rd }}$ Ed. Ed: FIM Craik \& TA Salthouse, pp. 1-54, Psychology Press, ISBN-13:978-0-8058-5990-4, New York

Ding, Q, Vaynman S, Akhavan M et al. (2006). Insulin-like growth factor I interfaces with brain-derived neurotrophic factor-mediated synaptic plasticity to modulate aspects of exercise-induced cognitive function. Neuroscience, 140:823-833

Driemeyer J, Boyke J, Gaser C, Buchel C, May A. Changes in gray matter induced learningrevisted. PLoS ONE, July 2008 Volume 3, Issue 7, e2669, www.plosone.org

Erickson KI, Prakash RS, Voss MW, Chaddock L, Hu L, Morris KS, White SM, Wójcicki TR, McAuley E, Kramer AF. (2009). Aerobic fitness is associated with hippocampal volume in elderly humans. Hippocampus, 19:1030-1039 doi:10.1002/hipo.20547 [published Online First: 2 January 2009]

Etnier JL, Nowell PM, Landers DM et al. (2006). A meta regression to examine the relationship between aerobic fitness and cognitive performance. Brain Research Reviews, 52:119-130

Ferris LT, William JS, Shen CL. (2007). The effect of acute exercise on serum brain-derived neurotrophic factor levels and cognition. Med Sci Sports Exerc, 39:728-734

Gunstad JP, Cohen RA,Tate DF, et al. (2008). Relationship between body mass index and brain volume in healthy adults. Int J Neurosci, 119:1582-1593 
Gustafson D, Lissner, L, Bengtsson, C et al. (2004). A 24-year follow-up of body mass index and cerebral atrophy. Neurology, 63:1876-1881

Haier RJ, Karama S, Leyba L, Jung RE. MRI assessment of cortical thickness and functional activity changes in adolescent girls following three months of practice on a visualspatial task. BMC Research Notes, 2009, 2:174 doi:10.1186/1756-0500-2-174. Published 1 September 2009

Haskell WL, Lee IM, Pate RR, et al. (2007). Physical activity and public health: updated recommendation from the American College of Sports Medicine and the American Heart Association. Med Sci Sports Exerc. 39:1423-34

Heyn P, Abreu BC, Ottenbacher KJ. (2004). The effects of exercise training on elderly persons with cognitive impairment and dementia: a meta analysis. Arch Phys Med Rehabil, 85:1694-1700

Jackson AS, Blair SN, Mahar MT, et al.(1990). Prediction of functional aerobic capacity without exercise testing. Med Sci Sports Exerc, 22:863-870

Kharti P, Blumenthal JA, Babyak MA, et al. (2001). Effects of exercise training on cognitive functioning among depressed older men and women. J Aging and Physical Activity, 9:43-57

Leasure JL, Jones M. (2008). Forced and voluntary exercise differentially affect brain and behavior. Neuroscience, 56:456-465

Liu Z, Zhu H, Marks, BL, Katz LM, Goodlett CB, Gerig G, Styner M. (2009). Voxel-wise group analysis of DTI. IEEE International Symposium on Biomedial Imaging (June 28July 1, 2009, Boston, MA) Proceedings of the 6th IEEE International Symposium on Biomedical Imaging: From Nano to Macro, NA-MIC, June 2009; 807-810

Lommatzsch M, Zingler D, Schuhbaeck K, et al. (2005). The impact of age, weight and gender on BDNF levels in human platelets and plasma. Neurobiology of Aging, 26:115-23

Madden DJ, Bennett HJ, Song AW. (2009). Cerebral white matter integrity and cognitive aging: contributions from diffusion tensor imaging. Neuropsychol Rev. Epub Ahead of Print: 25 August 2009. doi 10.1007/s11065-009-9113-2

Marks BL, Katz LM, Styner M, Smith JK. (2010). Aerobic Fitness and Obesity: Relationship to Cerebral White Matter Integrity in the Brain of Active and Sedentary Older Adults. British Journal of Sports Medicine, online first, doi:10.1136/bjsm.2009.068114

Marks BL, Madden DJ, Bucur B, et al. (2007). Role of aerobic fitness and aging on cerebral white matter integrity. Annals NY Acad Sci, 1097:171-174

Marks, BL, Katz, LM, Nunley, DC, Neelon, V, Daniel, P. (2000). Cerebral blood flow and cognitive function is maintained in aerobically active older adults. Circulation, Suppl, 102(18): 4198

Meriam Webster Free Dictionary, http://www.merriam-webster.com/dictionary/exercise, last accessed April 23, 2011

Nieman, D. (2011). Exercise Testing and Prescription, A Health Related Approach. 7th Edition, New York:McGraw Hill

Orlandi G, Murri L. (1996). Transcranial doppler assessment of cerebral flow velocity at rest and during voluntary movements in young and elderly subjects. Internat J Neurosci, 84(1-4):45-53 
Rahman, F, Smith K, Bullitt E, Marks B. (2008). Relationships between exercise and cerebral blood flow in older adults. American Society of Neuroradiology Conference, New Orleans, May 31 - June 5. Abstract ID: 6009535

Raji CA, Ho AJ, Parikshak NN, Becker JT, Lopez OL, Kuller LH, Hua X, Leow AD, Toga AW, Thompsom PM. (2009). Brain structure and obesity. Human Brain Mapp, Published Online First: 6 August 2009; doi: 10.1002/hbm.20870

Ross JS, Tkach J, Ruggieri PM, Lieber M, Lapresto E. (2003). The Mind's Eye: Functional MR imaging evaluation of golf motor imagery. American Journal of Neuroradiology, 24:1036-1044

Scheibel AB. (2009). Aging of the Brain, In: Handbook of the Neuroscience of Aging, Ed: PR Hoff $\mathcal{E}$ CV Mobbs, pp. 5-9, Academic Press, ISBN: 978-0-12-374898-0, New York:NY

Soreca I, Rosana C, Jennings R, et al. (2009). Gain in adiposity across 15 years is associated with reduced gray matter volume in healthy women. Psychosom Med, 71:485-490

Spirduso WW, Francis KL, MacRae PG. (2005). Physical Dimensions of Aging, 2nd Edition. Human Kinetics, Champaigne:IL

Stebbins GT, Murphy CM. (2009). Diffusion tensor imaging in Alzheimer's disease and mild cognitive impairment. Behav Neurol, 21(1):39-49

US Department of Health and Human Services (USDHHS). 2008 Physical Activity Guideline for Americans. ODPHP Pub. No. U0036, October 2008 www.health.gov/paguidleines last accessed April 23, 2011

US Department of Health and Human Services (USDHHS). National Institutes of Health Research Portfolio Online Reporting Tools (RePORT) http:/ / projectreporter.nih.gov/reporter.cfm) Last accessed April 25, 2011

Ward M, Carlsson CM, Trivedi MA, Sager MA, Johnson SC. (2005). The effect of body mass index on global brain volume in middle-aged adults: a cross-sectional study. BMC Neurol, 5:23 doi:10.1186/1471-2377-5-23. [Published Online 2005 December 2]

Vachet C, Bullitt E, Katz L, Marks B, Davis B, Styner M. (2009). UNC Elderly Brain Atlas, http://www.insight-journal.org/midas/item/view/2330 and http://www.insight-journal.org/midas/gallery In collection MIDAS/National Alliance for Medical Image Computing (NAMIC)/ NAMIC: Public Data Repository; NIH Neuroscience Roadmap Initiative

van Praag H, Christie BR, Sejnowski TJ, Gage FH. (1999). Running enhances neurogenesis, learning, and long-term potentiation in mice. Proc Natl Acad Sci, 96:13427-13431 


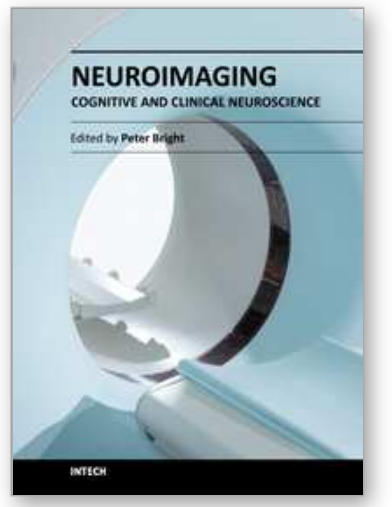

\author{
Neuroimaging - Cognitive and Clinical Neuroscience \\ Edited by Prof. Peter Bright
}

ISBN 978-953-51-0606-7

Hard cover, 462 pages

Publisher InTech

Published online 16, May, 2012

Published in print edition May, 2012

The rate of technological progress is encouraging increasingly sophisticated lines of enquiry in cognitive neuroscience and shows no sign of slowing down in the foreseeable future. Nevertheless, it is unlikely that even the strongest advocates of the cognitive neuroscience approach would maintain that advances in cognitive theory have kept in step with methods-based developments. There are several candidate reasons for the failure of neuroimaging studies to convincingly resolve many of the most important theoretical debates in the literature. For example, a significant proportion of published functional magnetic resonance imaging (fMRI) studies are not well grounded in cognitive theory, and this represents a step away from the traditional approach in experimental psychology of methodically and systematically building on (or chipping away at) existing theoretical models using tried and tested methods. Unless the experimental study design is set up within a clearly defined theoretical framework, any inferences that are drawn are unlikely to be accepted as anything other than speculative. A second, more fundamental issue is whether neuroimaging data alone can address how cognitive functions operate (far more interesting to the cognitive scientist than establishing the neuroanatomical coordinates of a given function - the where question).

\title{
How to reference
}

In order to correctly reference this scholarly work, feel free to copy and paste the following:

Bonita L. Marks and Laurence M. Katz (2012). MRI Techniques to Evaluate Exercise Impact on the Aging Human Brain, Neuroimaging - Cognitive and Clinical Neuroscience, Prof. Peter Bright (Ed.), ISBN: 978-953-510606-7, InTech, Available from: http://www.intechopen.com/books/neuroimaging-cognitive-and-clinicalneuroscience/mri-techniques-to-evaluate-exercise-impact-on-the-aging-human-brain

\section{INTECH}

open science | open minds

\section{InTech Europe}

University Campus STeP Ri

Slavka Krautzeka 83/A

51000 Rijeka, Croatia

Phone: +385 (51) 770447

Fax: +385 (51) 686166

www.intechopen.com

\section{InTech China}

Unit 405, Office Block, Hotel Equatorial Shanghai

No.65, Yan An Road (West), Shanghai, 200040, China

中国上海市延安西路65号上海国际贵都大饭店办公楼 405 单元

Phone: +86-21-62489820

Fax: +86-21-62489821 
(C) 2012 The Author(s). Licensee IntechOpen. This is an open access article distributed under the terms of the Creative Commons Attribution 3.0 License, which permits unrestricted use, distribution, and reproduction in any medium, provided the original work is properly cited. 